\title{
Projectile Electron Loss in Nitrogen
}

\author{
A. C. F. Santos ${ }^{1}$, W. S. Melo ${ }^{2}$, M. M. Sant'Anna ${ }^{1}$, G. M. Sigaud ${ }^{3}$, and E. C. Montenegro ${ }^{1}$ \\ 1 Instituto de Física, Universidade Federal do Rio de Janeiro, \\ Caixa Postal 68528, 21941-972 Rio de Janeiro, RJ, Brazil \\ ${ }^{2}$ Departamento de Física, Universidade Federal de Juiz de Fora, Juiz de Fora, MG, Brazil, 36036-330 \\ ${ }^{3}$ Departamento de Física, Pontifícia Universidade Católica do Rio de Janeiro, RJ, Brazil, Caixa Postal 38031
}

Received on 29 July, 2005

\begin{abstract}
The projectile electron loss channel plays an important role in modeling several processes connected to the penetration of swift ions in gases, such as radiation damage, energy loss, upper atmosphere studies, storage lifetimes of low-charge state heavy ions, etc. In this paper we have used recent measurements of projectile electron loss of $\mathrm{He}^{+}$ions in $\mathrm{N}_{2}$ together with previous data for higher charged ions in order to shed light on the role played by the projectile electron loss in the heating and ionization of Titans atmosphere.
\end{abstract}

Keywords: Stripping cross sections; Electron loss

\section{INTRODUCTION}

The understanding of the interactions between fast ions and the nitrogen molecule has been considerably favored recently due to its application to the study of Titans atmosphere [1], which is constituted of $95 \%$ of nitrogen, compared with the $78 \%$ in Earths atmosphere. The major energy supply to Titans upper atmosphere comes from solar radiation and ions $\left(\mathrm{H}^{+}, \mathrm{He}^{2+}\right)$, electrons and ions $\left(\mathrm{N}^{+}, \mathrm{O}^{+}\right.$, and $\left.\mathrm{O}^{2+}\right)$ from the magnestophere of Saturn and $\mathrm{H}^{+}, \mathrm{O}^{+}, \mathrm{OH}^{+}, \mathrm{H}_{2} \mathrm{O}^{+}, \mathrm{H}_{3} \mathrm{O}^{+}$, $\mathrm{H}_{2}^{+}$) from the dissociation of $\mathrm{H}_{2} \mathrm{O}^{+}$[2-4]. When a fast ion crosses Titans exosphere and interacts with the atmospheric gases, several collision products are produced which give rise to a chain of reactions among the atmospheric molecules. In addition, after receiving energy from the interaction, these atoms or molecules at the exosphere may escape the gravitational field, a process called atmospheric sputtering, which can be produced by solar wind ions or by ions from the magnetospheric plasma [5]. The proper modeling of the atmospheric sputtering requires cross sections associated to the formation of the various possible primary collisions products from all possible collision channels. Electron removal cross sections for both the target and the projectile, for collisions involving multielectron systems, have been measured for many decades. Despite the progress in the investigation of the role of the various collision channels from molecular targets, the data available in the literature is not enough to cover all cases. Concerning the electron loss channel, an effort to cover some of these gaps was made by Santos and DuBois [6], who have suggested an empirical scaling which applies to single and multiple electron loss from most projectiles.

The scope of this paper is twofold. First, total electron loss cross sections for $\mathrm{He}^{+}$on $\mathrm{N}_{2}$, measured by Melo et al. [7], are compared with data for other intermediate-to-fast projectiles on $\mathrm{N}_{2}$, using the scaling law suggested in ref. [6], in order to estimate the contribution to this channel from other ions of Saturns magnetosphere. Second, an estimate of the contribution from the projectile electron loss to the heating and ionization of Titans atmosphere is given. As these factors control the post-collisional chemistry, the average kinetic energy release of the $\mathrm{N}_{2}$ fragments is estimated. The projec- tile electron loss channel, despite being small compared with direct ionization, is a result of a harder collision which gives rise to a higher degree of fragmentation and thus a significant contribution to the atmosphere heating. Although the $\mathrm{He}^{+}$ ions studied in this work have velocities which do not occur in Titans atmosphere, the main conclusions can still be used to estimate the role of electron loss from other energetic ions present in Saturn's magnetosphere.

\section{SINGLE ELECTRON LOSS OF VARIOUS PROJECTILES BY N $\mathrm{N}_{2}$}

Figure 1 presents single electron loss cross sections of singly and multiply charged projectiles on $\mathrm{N}_{2}$ from refs. [712] scaled according the empirical rule from ref. [6]. $\mathrm{N}_{e f f}$ is defined as the effective number of projectile electrons that are available for removal. For one electron projectiles and for cases where the differences in the binding energies between the projectile shells or subshells are large, the choice of $\mathrm{N}_{e f f}$ is straighforward. On the other hand, the choice of $\mathrm{N}_{\text {eff }}$ can be quite arbitrary for the other cases. I is the ionization potential of the projectile and $\mathrm{I}_{o}$ the ionization potential of the $\mathrm{H}$ atom. The general behavior of the single electron loss from ref. [7] is very similar to our previous data for the Ar target [13]. This can be due, in part, to the fact that both targets have roughly the same number of outermost electrons. From 1.5 MeV and above, the single electron loss cross section can be expressed approximately as $\sigma_{12} \sim \mathrm{v}^{-1}$, where $\mathrm{v}$ is the velocity of $\mathrm{He}^{+}$, a result which is in agreement with CTMC calculations [11]. At high velocities, the first order Born approximation predicts $\mathrm{a} \mathrm{v}^{-2}$ dependence. From the scaled cross sections shown in Fig. 1, one can estimate the single electron cross sections of some Saturn magnetosphere ions. For $\mathrm{O}^{+}$ions, for instance, the maximum of the single electron loss cross sections is estimated to be $300 \mathrm{Mb}$.

\section{A. Energy deposition and kinetic energy release (KER) associated to electron loss}

The solar wind and Saturn's magnetospheric ions penetrate into the Titans exobase causing ionization and atmospheric 


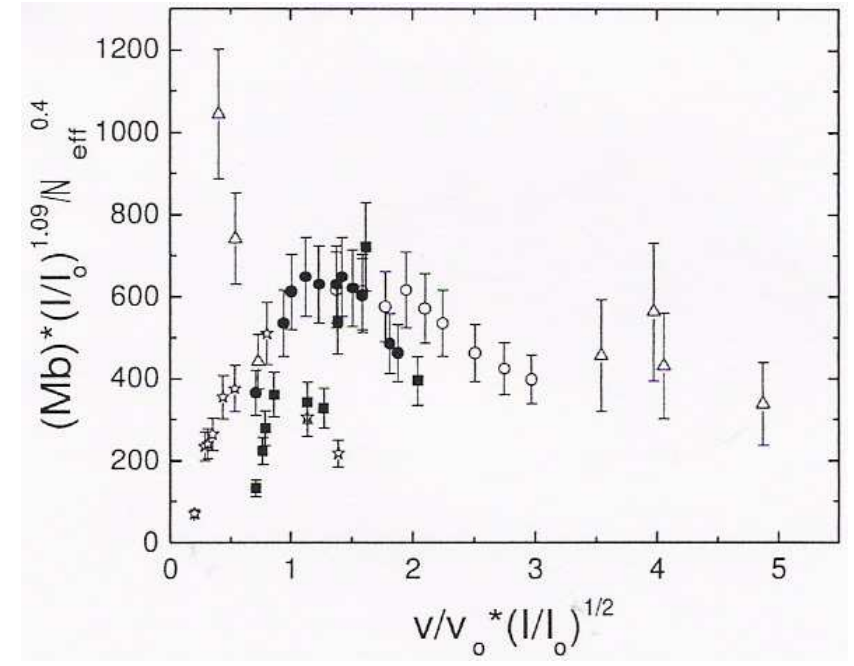

FIG. 1: Scaled cross sections plotted versus scaled velocities for single electron loss from singly and multiply charged ions on $\mathrm{N}_{2}$ (see text for details). Open circles $\mathrm{He}^{+}$, ref. [3]; closed circles, $\mathrm{He}^{+}$, ref. [4]; open triangles, $\mathrm{Ar}^{+}, \mathrm{Ar}^{2+}, \mathrm{Xe}^{3+}$, and $\mathrm{U}^{q+}$ from refs. [7, 8]; squares, $\mathrm{Xe}^{q+}$ ions (q=11-34) from ref. [10]; stars, $\mathrm{He}^{+}, \mathrm{Li}^{2+}, \mathrm{C}^{5+}$, and $\mathrm{O}^{7+}$ ions from ref. [9]. Here, $\mathrm{v} / \mathrm{v}_{o}$ is the projectile velocity in atomic units.

heating [14]. As mentioned above, the experimental determination of the Kinetic Energy Release (KER) in the fragmentation process is needed to model the dynamics of the atmosphere heating due to the post-collisional energy transfer of the fragments to the atmosphere. KER of the $\mathrm{N}_{2}$ fragments, associated with electron loss, due to $\mathrm{He}^{+}$impact, were determined from the shapes of the time-of-flight fragment peaks measured in coincidence with the projectile final charge state [7]. The measured processes related to the electron loss channel are:

$$
\begin{aligned}
& \mathrm{He}^{+}+\mathrm{N}_{2} \Rightarrow \mathrm{He}^{2+}+\mathrm{N}_{2}^{+}+2 \mathrm{e}-(\mathrm{a}) \\
& \mathrm{He}^{+}+\mathrm{N}_{2} \Rightarrow \mathrm{He}^{2+}+\mathrm{N}^{+}+\mathrm{N}+2 \mathrm{e}-(\mathrm{b}) \\
& \mathrm{He}^{+}+\mathrm{N}_{2} \Rightarrow \mathrm{He}^{2+}+\mathrm{N}_{2}^{2+}+3 \mathrm{e}-(\mathrm{c}) \\
& \mathrm{He}^{+}+\mathrm{N}_{2} \Rightarrow \mathrm{He}^{2+}+\mathrm{N}^{2+}+\mathrm{N}+3 \mathrm{e}-(\mathrm{d}) \\
& \mathrm{He}^{+}+\mathrm{N}_{2} \Rightarrow \mathrm{He}^{2+}+\mathrm{N}_{2}+\mathrm{e}-(\mathrm{e}) \\
& \mathrm{He}^{+}+\mathrm{N}_{2} \Rightarrow \mathrm{He}^{2+}+\mathrm{N}^{+}+\mathrm{N}^{+}+3 \mathrm{e}-(\mathrm{f})
\end{aligned}
$$

Processes ( $\mathrm{a}$ and $\mathrm{b}$ ) correspond to non-dissociative and dissociative loss-single ionization, respectively. Processes (c, $\mathrm{d}$ and f) correspond to loss-double ionization. Processes (b) and (c) could not be distinguished in the experiment described in ref. [7] because they have the same mass-to-charge ratio. Process (e) corresponds to projectile electron loss without target ionization. The experimental set-up used in ref. [7] cannot detect both fragments from process (f), consequently, process (f) is not distinguished from process (b).

Figure 2 shows the recoil ion spectra measured in coincidence with $\mathrm{He}^{2+}$ for an $\mathrm{N}_{2}$ target after $2.0 \mathrm{MeV} \mathrm{He}{ }^{+}$impact. The $\mathrm{N}_{2}^{+}$peak has a gaussian shape, which is a characteristic of ions with thermal momentum distribution and the calculated KER is essentially the same, irrespective to both projectile energy and final charge state [7]. For the $\mathrm{N}^{+}$fragment, the calculated KER is $1.0 \mathrm{eV}$, assuming that the larger contribu-

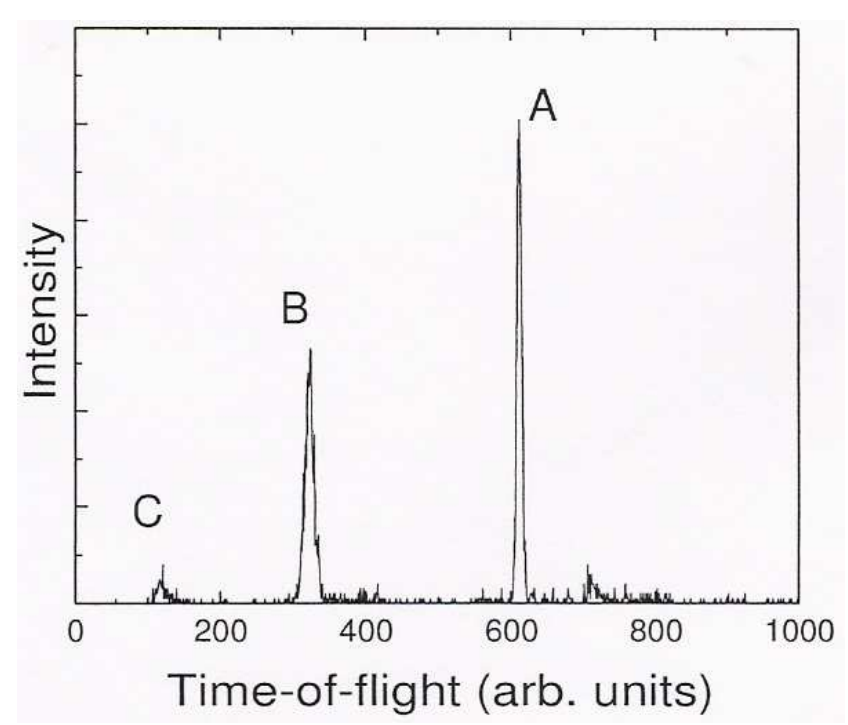

FIG. 2: Time-of-flight mass spectra of $\mathrm{N}_{2}$ recoils in coincidence with the $\mathrm{He}^{2+}$ at $2.0 \mathrm{MeV}$. Peak $\mathrm{A}, \mathrm{N}_{2}^{+}$(channel a); peak $\mathrm{B}, \mathrm{N}^{+}+\mathrm{N}_{2}^{2+}$ (channels $\mathrm{b}+\mathrm{c}+\mathrm{f}$ ); peak $\mathrm{C}, \mathrm{N}^{2+}$ (channel d)

tion to this peak comes from the $\mathrm{N}^{+}$fragment. For the $\mathrm{N}^{2+}$ fragment, the calculed KER is $3.3 \mathrm{eV}$.

The heating, $H$, due to ionic fragmentation above the Titan's thermospheric temperature, $T_{\text {therm }}$, is given by [15]

$$
H=\left\langle T_{o}\right\rangle-T_{\text {therm }}=\frac{1}{k}\left\langle U_{o}\right\rangle-T_{\text {therm }}
$$

where $k$ is the Boltzmann constant $\left(8.81 \times 10^{-5} \mathrm{eV} / \mathrm{K}\right),\left\langle U_{o}\right\rangle$ is the mean kinetic energy release, defined as

$$
\left\langle U_{o}\right\rangle=\frac{\sigma_{N_{2}^{+}} U_{N_{2}^{+}}+\sigma_{N^{+}} U_{N^{+}}+\sigma_{N^{2+}} U_{N^{2+}}}{\sigma_{N_{2}^{+}}+\sigma_{N^{+}}+\sigma_{N^{2+}}}
$$

where the labels $\mathrm{A}, \mathrm{B}$, and $\mathrm{C}$ are related to the corresponding peaks in Fig. 2. $T_{\text {therm }}$ is estimated to be $95 \mathrm{~K}[15] .\left\langle U_{o}\right\rangle$ is a slowly dependent function of the projectile energy and is estimated from Eq. 2 together with the data from ref. [7] as $0.5 \mathrm{eV}$, corresponding to a temperature of $3970 \mathrm{~K}$ and a heating $H=3830 \mathrm{~K}$ above Titans thermosphere temperature. It should be noted that the total electron loss cross section is comparable $(\sim 60 \%)$ to the total ionization cross section at high velocities and must be considered an important channel in producing heated fragments.

Schlachter et al. [16] calculated the contribution from the post-collisional interaction between the projectile and the recoiling ion making a parameterization of the recoil energies using the Classical Trajectory Monte Carlo method (CTMC). The energy transferred to the recoil ion in the direction tranverse to the projectile velocity follows a $\left(q_{1} q_{2}\right)^{2}$ rule, where $q_{1}$ and $q_{2}$ are the incoming projectile and recoil charge states, respectively. In the present single and double target ionization by $0.75-3.5 \mathrm{MeV} \mathrm{He}^{+}$projectiles impinging on $\mathrm{N}_{2}$ molecules, for impact parameters around $1 \mathrm{a}$.u, the transferred energy for the single target loss-ionization is between $1.1 \times$ 
$10^{-4} \mathrm{eV}-2.4 \times 10^{-4} \mathrm{eV}$, which is much smaller than the thermal kinetic energy $4 \times 10^{-2} \mathrm{eV}$ at $280 \mathrm{~K}$. In the case of double ionization, the transferred energy is $4.4-9.6 \times 10^{-4}$ $\mathrm{eV}$. One concludes that the recoil energies from the direct interaction with the projectile are negligible when compared to the kinetic energy release of the fragments, thus making a minor contribution to atmosphere heating.

\section{CONCLUSIONS}

Total electron loss cross sections of $\mathrm{He}^{+}$by $\mathrm{N}_{2}$ were compared with data for other intermediate-to-fast projectiles on $\mathrm{N}_{2}$ using the appropriate scaling law, in order to estimate the contribution to this channel from other ions of Saturns magnetosphere. In addition, the contribution from the projectile electron loss to the heating and ionization of Titans atmosphere was estimated. The projectile electron loss channel, although being a minor one when compared to direct ionization, is a result of a harder collision which gives rise to a higher degree of fragmentation and thus a significant contribution to atmospheric heating. $\mathrm{He}^{+}$ions with the velocities studied in this work do not occur in Titans atmosphere. However, the main conclusions of this work can still be used to estimate the role of electron loss from other energetic ions present in Saturn's magnetosphere.

\section{Acknowledgments}

The authors would like to express their gratitude to the Brazilian funding agencies FUJB (UFRJ), CAPES, CNPq, and FAPERJ.
[1] H. Luna, M. Michael, M. B. Shah, R. E. Johnson, C. J. Latimer, and J. W. McConkey, J. Geophys. Res. 108, 5033 (2002).

[2] L. E. Moore, M. Mendillo, I. C. F. Müller-Wodarg, and D. L. Murr, Icarus 172, 503 (2004).

[3] S. K. Atreya and T. M. Donahue, Icarus 24, 358 (1975).

[4] V. G. Anicich, J. Phys. Chem. Ref. Data 22, 1469 (1993).

[5] M. Michael, R. E. Johnson, F. Leblanc, M. Liu, J. G. Luhmann, and V. I. Shematovich, Icarus 175, 263 (2005).

[6] A. C. F. Santos and R. D. DuBois, Phys. Rev A 69, 042709 (2004).

[7] W. S. Melo, A. C. F. Santos, M. M. Sant'Anna, G. M. Sigaud, and E. C. Montenegro, to be published.

[8] L. I. Pivovar, V. M. Tubaev, and M. T. Novikov, Sov. Phys. JETP 14, 20 (1962).

[9] H. Knudsen, L. H. Andersen, H. K. Haugen, and P. Hvelplund, Phys. Scripta 26, 132 (1982).

[10] V. Horvat, R. L. Watson, K. E. Zaharakis, and Y. Peng, Nucl. Instr. Meth. Phys. Res. B 211, 495 (2003).

[11] R. D. DuBois, A. C. F. Santos, R. E. Olson, Th. Sthlker, F. Bosh , A. Brõuning-Demian, A. Gumberidze, S. Hagmann, C. Kozhuharov, R. Mann, A. Oršić Muthig, U. Spilman, S.
Tachenov, W. Barth, L. Dahl, B. Franzke, J. Glatz, L. Grning, S. Richter, D. Wilms, A. Krõmer, K. Ullman, and O. Jagutzki, Phys. Rev. A 68, 042701 (2003).

[12] R. D. DuBois, A. C. F. Santos, Th. Sthlker, F. Bosch, A. Brõuning-Demian, A. Gumberidze, S. Hagmann, C. Kozhuharov, R. Mann, A. Oršić Muthig, U. Spillmann, S. Tachenov, W. Bart, L. Dahl, B. Franzke, J. Glatz, L. Grning, S. Richter, D. Wilms, K. Ullmann, and O. Jagutzki, Phys. Rev A 70, 032712 (2004)

[13] M. M. Sant'Anna, W. S. Melo, A. C. F. Santos, G. M. Sigaud, and E. C. Montenegro, Nucl. Instr. Meth Phys. Res. B 99, 46 (1995).

[14] M. Michael and R. E. Johnson, Plan. and Space Sci. 53 (14-15): 1510-1514 (2005)

[15] H. M. Boechat-Roberty, S. Pilling, and A. C. F. Santos, to appear in Astron. \& Astrophys. (2005).

[16] A. S. Schlachter,K. H. Berker, H. F. Beyer, W. G. Graham, W. Groh, R. Mann, A. Müller, R. E. Olson, R. V. Pyle, J. W. Stearns, and J. A. Tanis, Phys. Scripta T3, 153 (1983). 